\title{
Markers of maternal and infant metabolism are associated with ventricular dysfunction in infants of obese women with type 2 diabetes
}

\author{
W. Todd Cade ${ }^{1}$, Philip T. Levy ${ }^{2}$, Rachel A. Tinius ${ }^{1}$, Mehgna D. Patel ${ }^{3}$, Swati Choudhry ${ }^{3}$, Mark R. Holland ${ }^{4}$, Gautam K. Singh ${ }^{2}$ \\ and Alison G. Cahill ${ }^{5}$
}

BACKGROUND: To test the hypothesis that infants born to obese women with pre-gestational type 2 diabetes mellitus (IBDMs) have ventricular dysfunction at 1 month that is associated with markers of maternal lipid and glucose metabolism.

METHODS: In a prospective observational study of IBDMs (OB+DM, $n=25)$, echocardiographic measures of septal, left (LV) and right ventricular (RV) function, and structure were compared at 1 month of age with those in infants born to $O B$ mothers without $D M(O B, n=24)$ and to infants born to non-OB mothers without DM (Lean, $n=23$ ). Basal maternal lipid and glucose kinetics and maternal plasma and infant (cord) plasma were collected for hormone and cytokine analyses.

RESULTS: RV, LV, and septal strain measures were lower in the OB+DM infants compared with those in other groups, without evidence of septal hypertrophy. Maternal hepatic insulin sensitivity, maternal plasma free-fatty-acid concentration, and cord plasma insulin and leptin most strongly predicted decreased septal strain in OB+DM infants.

CONCLUSION: IBDMs have reduced septal function at 1 month in the absence of septal hypertrophy, which is associated with altered maternal and infant lipid and glucose metabolism. These findings suggest that maternal obesity and DM may have a prolonged impact on the cardiovascular health of their offspring, despite the resolution of cardiac hypertrophy.

S ince the mid-1940s, cardiac hypertrophy at birth, particularly in the septum, has been a well-known phenomenon in infants born to women with pre-gestational type 2 diabetes mellitus (IBDM) (1). This hypertrophy is thought to be transient, often resolving within 2 weeks to 6 months of age (1-4). Emerging evidence suggests that ventricular function during gestation and at birth is impaired in IBDMs, even in the absence of cardiac hypertrophy $(5,6)$. Although unclear from the current literature, early-life ventricular abnormalities may predispose these children to developing overt cardiac dysfunction as adults, or as other cardiovascular risk factors emerge.

Mechanisms for ventricular contractile abnormalities in IBDMs remain obscure. Some data $(5,7,8)$, but not all (9), suggest that maternal and infant glycemia is associated with impaired cardiac function. Recent evidence points to a possible relationship between maternal adipose tissue/lipid metabolism and adverse cardiac function in the offspring $(6,10)$. Additional findings demonstrate the association of maternal lipid metabolism with elevated infant birth weight in diabetic pregnancy (11-13). None of these previous studies have separated the effects of maternal obesity and diabetes mellitus on cardiac function in infants, nor have they examined the predictive value of maternal lipid and glucose metabolism on infant ventricular function. Furthermore, advances in neonatal cardiac imaging permit a more comprehensive assessment of ventricular function in neonates that could not be previously obtained with conventional imaging (14).

Therefore, the primary objective of this study was to compare left (LV) and right ventricular (RV) functions in infants born to the following three groups, using conventional and two-dimensional (2D) quantitative echocardiography: (i) obese women with pre-gestational type 2 diabetes mellitus $(\mathrm{OB}+\mathrm{DM})$; (ii) obese women without diabetes mellitus $(\mathrm{OB})$; and (iii) non-obese women without diabetes mellitus (Lean). The secondary objective was to examine the indices of maternal lipid metabolism as predictors of infant ventricular function in this cohort. Our hypothesis was that ventricular functional abnormalities will be greatest in infants born to obese women with DM and would be associated with markers of maternal lipid metabolism.

\section{METHODS}

This was a prospective observational study of three groups of women who were receiving prenatal care at the Women's Health Center at 
Barnes-Jewish Hospital/Washington University School of Medicine in St Louis between May 2011 and December 2013, and their neonates. Infants were stratified into three separate groups based on maternal body mass index and diabetic status: infants born to (i) obese women with pre-gestational, type $2 \mathrm{DM}$, requiring insulin (White Class B or C $(\mathrm{OB}+\mathrm{DM})$ ); (ii) obese women without $\mathrm{DM}$ or insulin resistance (no pre-gestational or gestational and normal $50 \mathrm{~g}$ glucose challenge (OB)); and (iii) non-obese controls without DM (Lean). Obesity was defined as pre-pregnancy body mass index between 30 and $45 \mathrm{~kg} / \mathrm{m}^{2}$. Women with pre-gestational DM were on insulin therapy, and had $\mathrm{HbAlC} \leq 8(183 \mathrm{mg} / \mathrm{dl}, 64 \mathrm{mmol} / \mathrm{mol})$ for more than 3 months before pregnancy as previously described (13). Exclusion criteria for all women included the following: (i) multiple gestation pregnancy; (ii) inability to provide voluntary informed consent; (iii) current self-reported use of illegal drugs (cocaine, methamphetamine, and opiates); (iv) current smoker who did not consent to cessation; (v) current usage of daily medications by class: corticosteroids, beta-blockers (known to affect lipid metabolism), or antipsychotics (known to alter insulin resistance and metabolic profiles); and (vi) known fetal anomalies. For women without DM, exclusion criteria included the following: (i) diagnosis or history of gestational diabetes or abnormal $50 \mathrm{~g}$ glucose challenge between 24 and 28 weeks; (ii) pre-pregnancy DM; or (iii) prior macrosomic $(>4,000 \mathrm{~g})$ infant. This study was approved by the Human Research Protection Office at Washington University School of Medicine (IRB\#201012828, NCT\#01346527).

\section{Assessment of Cardiac Structure and Function}

A transthoracic complete M-mode, 2D and Doppler echocardiographic examination was performed at 1 month of age with a commercially available ultrasound imaging system (Vivid 7 and 9; General Electric Medical Systems, Milwaukee, WI). The timing of the echocardiographic study was carefully selected to take place at 1 month of age to avoid the early postnatal period of cardiopulmonary instability with a patent ductus arteriosus and rapidly decreasing pulmonary vascular resistance. All of the infants were clinically healthy at birth and were discharged home with their mothers. None of the infants in this study had evidence of a patent ductus arteriosus or altered pulmonary hemodynamics at 1 month of age. All infants were stable in room air at the time of the study. All exams were performed $2 \mathrm{~h}$ after feedings. One designated pediatric cardiac sonographer, blinded to the maternal and infant clinical and demographic data and experienced in acquiring images for conventional and 2D speckle tracking echocardiographic (2DSTE) analysis, obtained all the echocardiographic images using a transducer (7.5-12 MHz) center-frequency-phased array probe and optimized to visualize the myocardial walls (15). The echocardiographic images were acquired according to the guidelines of the American Society of Echocardiography (16) and with a previous validated protocol in decubitus position during the restful period without changing the position of the infant or disturbing the hemodynamic condition to minimize heart rate and respiratory variation during the image acquisition $(15,17)$.

Cardiac structure. Relative wall thickness and LV mass index (LVMI, Devereux formula) were calculated using M-mode imaging of the LV in the parasternal short-axis view, and LVMI was indexed to height ${ }^{2.7}\left(\mathrm{~g} / \mathrm{m}^{2.7} ;\right.$ (refs 16,18)). Using M-mode imaging, interventricular septal wall thickness was measured in the parasternal long-axis view at the level of the tip of the mitral valve midway between the apex and the crux of the heart. The RV linear dimensions (inflow at base and mid-cavity, length, and tricuspid valve annulus) were measured from the apical four-chamber view. The RV linear dimensions at the proximal and distal outflow were measured from the parasternal short-axis view.

Cardiac function. Conventional indices of LV function, shortening fraction, and biplane LV ejection fraction were measured according to the guidelines of the American Society of Echocardiography
$(16,19,20)$. Myocardial mechanics were analyzed by the quantification of LV, RV, and septal longitudinal strain (\%) and strain rate $(\% / s)$ by 2DSTE, an emerging quantitative echocardiographic technique to characterize ventricular function in neonates (14). The strain parameters were acquired using previously validated image acquisition and data analysis protocols from our laboratory $(15,17)$. A frame rate to heart rate ratio between 0.7 and 0.9 frames/s per bpm was utilized to optimize myocardial speckle tracking and mechanical event timing (21). LV global longitudinal strain (LV GLS, $\%$ ) and systolic, early, and late diastolic GLS rates (LV GLSRs, GLSRe, and GLSRa (\%/s), respectively) were calculated by averaging all values of the regional peak longitudinal strain obtained from 17 segments in two-chamber, apical long-axis, and apical four-chamber views (22). Peak strain was measured as end-systolic strain at the closure of the aortic valve (23). RV GLS (RV GLS, \%) was measured from an RV-focused apical four-chamber view and was calculated by averaging all values of the regional peak longitudinal strain obtained from six segments. LV and RV segmental longitudinal strain (SLS, $\%)$ were measured at the apical-, mid-, and basal-ventricular levels in the RV and LV free walls. Septal GLS and GLSRs, GLSRe, and GLSRa measures were calculated by averaging all values of the SLS obtained from nine segments in the two-chamber, apical long-axis, and apical four-chamber views along the septal wall. A lower magnitude of strain $(\%)$ and strain rate $(\% / s)$ indicates worse ventricular function. A single observer (P.L.), who was blinded to the maternal and infant clinical and metabolic values, analyzed the strain imaging using vendor-customized commercially available software (EchoPAC; General Electric Medical Systems, Waukesha, WI, version 110.0.x). We have previously demonstrated reliable intra- and inter-observer reproducibility of strain imaging in neonates $(15,17,24)$.

\section{Lipid and Glucose Metabolism Studies}

All pregnant women underwent basal lipid and glucose kinetic studies (using stable isotope tracer methodology), and a subset $(n=49)$ also underwent hyperinsulinemic-euglycemic clamp during gestation weeks 32-36 as previously described (13). Maternal plasma at 32-36 weeks' gestation and infant (cord) plasma at birth were collected for hormone and cytokine analyses as previously described (13).

\section{Statistical Analyses}

Normally distributed demographics, plasma metabolite, hormone variables, and echocardiographic variables between groups were examined by one-way ANOVA, and group differences were compared through post hoc testing using Tukey honestly significant difference testing. Non-normally distributed variables determined by the Shapiro-Wilk test were examined by $\chi^{2}$ and independentsamples Kruskal-Wallis one-way ANOVA. Relationships between outcome variables were examined using univariate analysis (Pearson's Product Correlation Coefficient), and standardized linear regression analysis with Bonferroni adjustment for multiple correlations was used to refine the ability to predict LV, RV, and septal global and regional deformation strain combining multiple metabolic measures. Univariate analysis was utilized to determine the best predictors to enter in the model (significant correlation $>0.4$ ), and then backward step-wise regression was performed. Statistical significance was considered at $P<0.05$. Because of the lack of data regarding the relationship between maternal and infant lipid and glucose metabolism with postnatal ventricular function, and the exploratory nature of this study, we used data from our previous work in nongravid insulin-resistant and control participants and data from our deformation studies to estimate the sample size, assuming an alpha of 0.05 , where 20 subjects per group would provide $99 \%$ power to detect $20 \%$ differences in echocardiographic measures between groups $(13,24,25)$. All statistical analyses were performed using SPSS (IBM, Armonk, NY).

\section{RESULTS}

Seventy-nine women were enrolled and studied at 32-36 weeks' gestation. Of the neonates from 79 mothers 
enrolled in the original study (13), 72 neonates returned at 1 month of age ( $30 \pm 5$ days) and received conventional and speckle-tracking echocardiograms between May 2011 and December 2013. Infants were stratified into the following three separate groups: (i) $\mathrm{OB}+\mathrm{DM}, n=25$; (ii) $\mathrm{OB}, n=24$; and (iii) Lean, $n=23$. The infants included were from a previous study reported by our group; however, their echocardiographic data were not initially analyzed in the study (13). There was a statistical difference in the gestational ages at birth between the $\mathrm{OB}+\mathrm{DM}(37 \pm 2$ weeks $)$ and the $\mathrm{OB}$ $(39 \pm 2$ weeks) and Lean $(39 \pm 1$ weeks) groups, but after adjusting for birthweight, gender, and postnatal age at the time of echocardiography, univariate correlations did not reveal relationships between the echocardiographic indices and gestational age between the groups. Table 1 displays the demographic, clinical, and metabolic characteristics of the study population.

\section{Assessment of Cardiac Structure and Function}

Ventricular structure. LV mass, LV mass index, relative wall thickness, and interventricular septal wall thickness were not different between the three groups. RV major dimension was only significantly higher between the $\mathrm{OB}+\mathrm{DM}$ and $\mathrm{OB}$ groups $(P<0.05$; Table 2$)$.

Ventricular systolic function. The results of the ventricular functional analyses are provided in Table 2. There were no differences in conventional measures of LV systolic function (ejection fraction and shortening fraction) among groups. Septal GLS was significantly lower in $\mathrm{OB}+\mathrm{DM}$ compared with both the OB and Lean groups $(P<0.005$ for both). Septal SLS displayed a preserved normal apex-to-base gradient pattern in all three groups $(P<0.05)$, but SLS at the apex and mid-level of the septum was significantly lower in $\mathrm{OB}+\mathrm{DM}$ compared with that in $\mathrm{OB}$ and Lean groups $(P<0.002$ for both). LV GLS was significantly lower in the $\mathrm{OB}+\mathrm{DM}$ vs. the Lean group $(P<0.05)$. LV SLS also displayed a preserved normal apex-tobase gradient pattern $(P<0.05)$, but LV SLS was significantly lower at the basal- and mid-ventricular levels in the $\mathrm{OB}+\mathrm{DM}$ group vs. that in the Lean group $(P<0.05)$. RV GLS was significantly lower in the $\mathrm{OB}+\mathrm{DM}$ group vs. that in the Lean group $(P<0.05)$. RV SLS displayed a preserved normal base-toapex gradient pattern $(P<0.05)$, with no differences in values in any of the three groups. RV and LV systolic strain rate patterns were all similar among the groups.

Ventricular diastolic function. The feasibility of GLSRe and GLSRa was $93 \%$, as 7 out of 72 patients had fused early- and late-strain curves and were excluded from the analysis. LV GLSRe and GLSRa were not different between the groups; however, both RV and Septal GLSRe were significantly lower in the OB+DM group vs. the Lean group $(P<0.001)$.

Relationships between Maternal Lipid and Glucose Kinetics with Ventricular Deformational Indices

Using univariate analysis in $\mathrm{OB}$ and $\mathrm{OB}+\mathrm{DM}$ women only $(n=49)$, several maternal and infant variables were tested for association with LV, RV, and septal GLS. Variables found to have an association with septal GLS $(\mathrm{R} \geq 0.40, P<0.05)$ included maternal age, endogenous glucose production per unit of insulin during the baseline period and during hyperinsulinemia, glucose rate of disappearance during hyperinsulinemia (i.e., insulin sensitivity), suppression of endogenous glucose production with hyperinsulinemia, and plasma free-fatty-acid concentration during hyperinsulinemia. Infant variables with a correlation with septal SLS $(R \geq 0.40, P<0.05)$ included cord plasma insulin, C-peptide, leptin, and infant birth weight. Using these variables in a backward stepwise regression analysis (multicollinearity: tolerance $=0.52-0.78$, variance inflation factor $=1.3-1.9)$, a model including maternal plasma free-fatty-acid concentration during hyperinsulinemia, suppression of endogenous glucose production with hyperinsulinemia, infant birth weight and cord plasma insulin, and leptin most strongly predicted a decreased septal strain in the $\mathrm{OB}+\mathrm{DM}$ group (adjusted $R^{2}=0.62$ ). None of the maternal or infant lipid and glucose metabolism variables were associated with LV or RV strain measures.

\section{DISCUSSION}

In this study we utilized an emerging non-invasive echocardiographic modality, deformation imaging by 2DSTE, to quantitatively characterize ventricular performance and assess early-stage, disease-related cardiac changes in IBDMs at 1 month of age. The principal and novel finding of this study is that IBDMs have reduced septal strain (septal function) in the absence of septal hypertrophy that was associated with alterations in maternal lipid and maternal and infant glucose metabolism. In addition, IBDMs had decreased RV and LV deformation values with relatively preserved ventricular structure.

Pre-gestational diabetes has an effect on early embryonic development that may alter the cardiac morphogenesis during gestation and may extend to the neonatal period, exposing IDBMs to various degrees of myocardial damage (5). The high levels of glucose in the maternal blood will cause fetal hyperglycemia through the placenta, leading to fetal cardiomyocyte injury directly, with excessive apoptosis of myocardial cells (26). Pre-gestational type $2 \mathrm{DM}$ is also characterized by hyperlipidemia $(6,27)$. As the pregnancy progresses, the excessive availability of lipids and higher maternal free-fattyacid concentration might lead to increased fatty-acid delivery to the fetus, resulting in lipid accumulation within the fetal cardiomyocyte $(6,26)$. The fetal heart adapts to the hyperlipidemia by increasing the expression of fatty-acidmetabolizing proteins, thereby increasing the reliance on fatty acids as energy $(6,26)$. This adapting heart is initially able to maintain cardiac output under these conditions. However, the continued exposure of the fetal heart to this metabolic environment eventually leads to apoptosis, fibrosis, and contractile dysfunction (6). In this study we found that several maternal and infant metabolic variables had associations with decreased septal strain in infants born to obese and 


\section{Cardiac dysfunction in infants of DM 1 Articles}

Table 1. Maternal and infant demographics and plasma metabolites

\begin{tabular}{|c|c|c|c|c|}
\hline & Lean $(n=23)$ & $\mathrm{OB}(n=24)$ & $\mathrm{OB}+\mathrm{DM}(n=25)$ & Fratio $P$ value \\
\hline \multicolumn{5}{|l|}{ Maternal variables } \\
\hline Age (years) & $23 \pm 3$ & $25 \pm 5$ & $30 \pm 6^{*}, \dagger$ & $<0.001$ \\
\hline African-American & $20(87 \%)$ & $22(92 \%)$ & $19(76 \%)$ & \\
\hline Caucasian & $3(13 \%)$ & $2(8 \%)$ & $5(20 \%)$ & \\
\hline Weight (kg) & $69.9 \pm 7.9$ & $112.2 \pm 21.3$ & $127.6 \pm 21.9^{*} \dagger$ & $<0.001$ \\
\hline Gravida (\#) & $2 \pm 1$ & $3 \pm 2$ & $4 \pm 3^{\dagger}$ & 0.05 \\
\hline \multicolumn{5}{|l|}{ Prenatal visits $(n, \%)$} \\
\hline $1-5$ Visits & $(2,8)$ & $(2,8)$ & $(1,4)$ & \\
\hline Total cholesterol (mg/dl) & $206.0 \pm 33.6$ & $197.8 \pm 35.6$ & $178.5 \pm 38.4^{\dagger}$ & 0.03 \\
\hline HDL-cholesterol (mg/dl) & $71.3 \pm 17.7^{\ddagger}$ & $57.7 \pm 13.6$ & $57.0 \pm 15.6$ & 0.003 \\
\hline LDL-cholesterol (mg/dl) & $105.5 \pm 30.0$ & $108.4 \pm 30.7$ & $86.0 \pm 33.5^{\dagger}$ & 0.03 \\
\hline Leptin $(\mu \mathrm{g} / \mathrm{l})$ & $20.5 \pm 6.3$ & $49.2 \pm 20.3^{* *}$ & $53.9 \pm 38.6^{*}, \dagger$ & $<0.001$ \\
\hline IL-6 (pg/ml) & $2.8 \pm 1.0$ & $3.6 \pm 1.8$ & $4.1 \pm 2.1^{\dagger}$ & 0.03 \\
\hline IGF-1 (ng/ml) & $313.8 \pm 92.6$ & $280.1 \pm 103.8$ & $394.1 \pm 190.4^{*}$ & 0.02 \\
\hline \multicolumn{5}{|l|}{ Infantile variables } \\
\hline Gestational age (weeks) & $38 \pm 1$ & $39 \pm 2$ & $37 \pm 2^{*} \dagger$ & $<0.001$ \\
\hline Birth length $(\mathrm{cm})$ & $50.3 \pm 2.6$ & $50.8 \pm 2.2$ & $50.6 \pm 2.9$ & 0.73 \\
\hline Ponderal index & $24.1 \pm 2.3$ & $25.7 \pm 2.2$ & $27.5 \pm 3.5^{\dagger}$ & 0.01 \\
\hline APGAR-1 min & $8 \pm 1$ & $7 \pm 2$ & $6 \pm 3^{\dagger}$ & 0.005 \\
\hline APGAR-5 min & $9 \pm 1$ & $9 \pm 1$ & $8 \pm 2^{*}, \dagger$ & 0.004 \\
\hline \multicolumn{5}{|l|}{ Cord blood } \\
\hline Glucose (mg/dl) & $93.1 \pm 27.0$ & $78.9 \pm 14.4$ & $86.9 \pm 28.3$ & 0.09 \\
\hline Insulin $(\mu \mathrm{U} / \mathrm{ml})$ & $11.8 \pm 7.4$ & $10.9 \pm 6.9$ & $27.8 \pm 24.1^{*}, \dagger$ & $<0.001$ \\
\hline C-peptide (ng/ml) & $0.87 \pm 0.54$ & $1.18 \pm 0.81$ & $1.83 \pm 1.13^{*, \dagger}$ & $<0.001$ \\
\hline HOMA-IR & $2.8 \pm 2.0$ & $2.2 \pm 1.7$ & $6.0 \pm 6.2^{*, \dagger}$ & 0.001 \\
\hline IGF-1 (ng/ml) & $61.2 \pm 30.2$ & $52.4 \pm 19.7$ & $70.5 \pm 43.5$ & 0.49 \\
\hline $\mathrm{FFA}(\mathrm{mEq} / \mathrm{l})$ & $0.14 \pm 0.10$ & $0.15 \pm 0.10$ & $0.16 \pm 0.06$ & 0.29 \\
\hline Leptin $(\mu \mathrm{g} / \mathrm{l})$ & $11.2 \pm 6.9$ & $13.3 \pm 9.1^{* *}$ & $30.9 \pm 25.1^{*, \dagger}$ & $<0.001$ \\
\hline IL-6 (pg/ml) & $9.1 \pm 10.4$ & $10.0 \pm 12.1$ & $10.5 \pm 13.2$ & 0.83 \\
\hline
\end{tabular}

OB, obese; DM, diabetes mellitus; FFA, plasma free-fatty acid; IGF-1, plasma insulin-like growth factor-1; IL-6, interleukin 6; NICU, neonatal intensive care unit. Values are means \pm SD.

Post hoc analysis: ${ }^{*} P<0.05, \mathrm{OB}+\mathrm{DM}$ vs. $\mathrm{OB} ;{ }^{*} P<0.05, \mathrm{OB}$ vs. Lean; ${ }^{+} P<0.05, \mathrm{OB}+\mathrm{DM}$ vs. Lean. 


\section{Articles | Cade et al.}

Table 2. Neonatal echocardiographic parameters

\begin{tabular}{|c|c|c|c|c|}
\hline Variable & Lean $(n=23)$ & Obese $(n=24)$ & Obese+DM $(n=28)$ & F-ratio $P$ value \\
\hline HR (bpm) & $155 \pm 17(118-186)$ & $160 \pm 17(133-196)$ & $158 \pm 23(117-226)$ & 0.72 \\
\hline \multicolumn{5}{|l|}{ LV structure } \\
\hline LVM (g) & $10.6 \pm 3.0(7.1-20.0)$ & $11.6 \pm 3.4(6.8-19.6)$ & $11.6 \pm 2.8(7.8-19.3)$ & 0.38 \\
\hline LVMI & $46.3 \pm 11.0(31.0-80.1)$ & $50.9 \pm 13.0(34.9-78.4)$ & $49.5 \pm 11.5(33.5-80.3)$ & 0.42 \\
\hline RWT (mm) & $0.30 \pm 0.08(0.18-0.47)$ & $0.36 \pm 0.08(0.18-0.54)$ & $0.35 \pm 0.12(0.17-0.72)$ & 0.14 \\
\hline \multicolumn{5}{|l|}{ LV systolic function } \\
\hline $\mathrm{EF}(\%)$ & $64 \pm 6(52-71)$ & $64 \pm 9(51-81)$ & $64 \pm 7(56-80)$ & 0.92 \\
\hline SF (\%) & $34 \pm 4(28-43)$ & $38 \pm 5(30-47)$ & $36 \pm 5(28-45)$ & 0.06 \\
\hline GLS (\%) & $-19.2 \pm 1.5(-15.7-21.0)$ & $-18.0 \pm 2.1(-12.5-21.5)$ & $-17.5 \pm 1.9(-15.0-20.2)^{*}$ & 0.008 \\
\hline GLSRs (1/s) & $-1.6 \pm 0.2(-1.1-2.1)$ & $-1.6 \pm 0.2(-1.1-2.1)$ & $-1.6 \pm 0.3(-1.2-2.0)$ & 0.77 \\
\hline SLS base (\%) & $-19.5 \pm 3.0(-9.5-23.2)$ & $-16.7 \pm 3.4(-16.0-28.0)^{* *}$ & $-17.9 \pm 2.3(-13.6-22.4)^{*}$ & 0.01 \\
\hline SLS mid (\%) & $-19.2 \pm 2.0(-16.1-24.0)$ & $-16.6 \pm 3.0(-8.9-21.4)^{* *}$ & $-17.9 \pm 2.9(-9.2-22.4)^{*}$ & 0.01 \\
\hline SLS apex (\%) & $-22.7 \pm 2.2(-16.8-26.3)$ & $-21.6 \pm 3.7(-14.5-28.6)$ & $-21.2 \pm 3.3(-12.9-25.7)$ & 0.23 \\
\hline \multicolumn{5}{|l|}{ LV diastolic function } \\
\hline GLSRe (1/s) & $2.9 \pm 0.6(2.2,4.5)$ & $2.7 \pm 0.7(1.7,3.6)$ & $2.5 \pm 0.6(1.7,3.5)$ & 0.11 \\
\hline GLSRa (1/s) & $2.2 \pm 0.7(0.6,3.6)$ & $2.1 \pm 0.7(1.3,3.6)$ & $1.9 \pm 0.7(0.4,2.9)$ & 0.17 \\
\hline \multicolumn{5}{|l|}{ RV structure } \\
\hline RV length $(\mathrm{mm})$ & $3.0 \pm 0.2(2.9,3.3)$ & $2.9 \pm 0.2(2.7,3.2)$ & $3.0 \pm 0.2(2.6,3.4)$ & 0.19 \\
\hline RV major (mm) & $1.8 \pm 0.2(1.5,2.3)$ & $1.8 \pm 0.1(1.6,2.3)$ & $1.9 \pm 0.2(1.6,2.3)^{\dagger}$ & 0.04 \\
\hline RV minor (mm) & $1.5 \pm 0.1(1.4,1.9)$ & $1.5 \pm 0.1(1.3,1.7)$ & $1.5 \pm 0.1(1.3,1.7)$ & 0.31 \\
\hline \multicolumn{5}{|l|}{ RV systolic function } \\
\hline GLS (\%) & $-22.1 \pm 2.3(-18.2,-21.0)$ & $-21.4 \pm 3.5(-13.8,-26.8)$ & $-19.5 \pm 3.9(-7.8,-25.8)^{*}$ & 0.03 \\
\hline GLSRs (1/s) & $-2.0 \pm 0.3(-1.7,-2.6)$ & $-2.0 \pm 0.3(-1.2,-2.9)$ & $-1.9 \pm 0.4(-1.4,-2.9)$ & 0.29 \\
\hline SLS base (\%) & $-30.3 \pm 5.3(-18.7,-40.5)$ & $-29.2 \pm 7.1(-16.4,-40.3)$ & $-27.4 \pm 8.0(-11.2,-44.4)$ & 0.36 \\
\hline SLS mid (\%) & $-27.1 \pm 4.6(-16.1,-35.0)$ & $-25.9 \pm 7.1(-1.9,-35.2)$ & $-24.5 \pm 7.7(-3.2,-38.8)$ & 0.41 \\
\hline SLS apex (\%) & $-19.8 \pm 6.0(-9.6,-29.2)$ & $-19.6 \pm 6.3(-6.3,-30.9)$ & $-18.2 \pm 6.5(-5.3,-32.9)$ & 0.61 \\
\hline \multicolumn{5}{|l|}{ RV diastolic function } \\
\hline GLSRe (1/s) & $3.1 \pm 0.7(2.2,4.5)$ & $2.9 \pm 0.8(1.4,4.5)$ & $2.4 \pm 0.7(1.1,3.4)^{*}, \dagger$ & 0.008 \\
\hline GLSRa (1/s) & $2.5 \pm 0.9(0.3,3.8)$ & $2.1 \pm 0.8(0.3,3.5)$ & $2.1 \pm 0.8(0.6,3.5)$ & 0.12 \\
\hline \multicolumn{5}{|l|}{ Septal structure } \\
\hline SWT (mm) & $0.41 \pm 0.06(0.36-0.58)$ & $0.43 \pm 0.06(0.27-0.51)$ & $0.42 \pm 0.06(0.27-0.55)$ & 0.47 \\
\hline \multicolumn{5}{|c|}{ Septal systolic function } \\
\hline GLS (\%) & $-19.1 \pm 1.2(-16.5,-20.9)$ & $-19.2 \pm 2.7(-18.9,-21.2)$ & $-17.7 \pm 2.4(-13.2,-21.9)^{*}, \dagger$ & 0.005 \\
\hline GLSRs (1/s) & $-1.7 \pm 0.2(-1.3,-2.2)$ & $-1.8 \pm 0.3(-1.3,-2.5)$ & $-1.8 \pm 0.4(-1.2,-2.6)$ & 0.65 \\
\hline SLS apex (\%) & $-22.5 \pm 1.9(-19.1,-25.5)$ & $-23.0 \pm 6.3(-6.3,-28.6)$ & $-20.6 \pm 2.9(-11.0,-26.2)^{*, \dagger}$ & 0.002 \\
\hline SLS mid (\%) & $-18.0 \pm 1.6(-15.3,-35.0)$ & $-18.5 \pm 1.5(-15.2,-21.0)$ & $-16.5 \pm 2.6(-8.1,-22.4)^{*}, \dagger$ & 0.002 \\
\hline \multicolumn{5}{|c|}{ Septal diastolic function } \\
\hline GLSRe (1/s) & $3.0 \pm 0.4(2.0,3.7)$ & $2.8 \pm 0.6(1.6,4.0)$ & $2.2 \pm 0.4(1.3,3.6)^{*}, \dagger$ & 0.004 \\
\hline GLSRa (1/s) & $2.7 \pm 0.6(0.9,3.3)$ & $2.6 \pm 0.5(0.9,3.6)$ & $2.6 \pm 0.8(0.8,3.3)$ & 0.67 \\
\hline
\end{tabular}

bpm, beat per minute; EF, ejection fraction; GLS, global longitudinal strain; GLSRa, late global longitudinal diastolic strain rate; GLSRe, early global longitudinal diastolic strain rate; GLSRs, global longitudinal systolic strain rate; HR, heart rate; Lean, infants born to women without obesity or diabetes; LV, left ventricle; LVM, left ventricular mass measured by M-mode echocardiography; LVMI, left ventricular mass index; Obese, infants born to women with obesity but not diabetes; Obese+DM, infants born to women with obesity and diabetes; RWT, relative wall thickness; SF, shortening fraction; SWT, septal wall thickness.

Values are mean \pm SD. Range provided in parentheses.

${ }^{*} P<0.05 \mathrm{OB}+\mathrm{DM}$ vs. Lean.

${ }^{* *} P<0.05 \mathrm{OB}$ vs. Lean.

$* * P<0.05 \mathrm{OB}+\mathrm{DM}$ vs. OB. 


\section{Cardiac dysfunction in infants of DM $\quad$ Articles}

diabetic women, including maternal hepatic insulin sensitivity, maternal plasma free-fatty-acid concentration, and cord blood insulin and leptin.

The complex interaction between excessive glucose and lipid metabolism affects the heart of IBDMs in multiple ways, including causing cardiac malformations, hypertrophic cardiomyopathy (even with good maternal glycemic control), and functional impairment (even in the absence of structural changes) (28). The most common cardiac pathology in IBDMs is asymmetrical septal hypertrophy (28-30). Myocardial mass is thought to increase because of the presence of higher levels of fetal insulin during gestation that can lead to septal wall dysfunction immediately after birth. Septal wall dysfunction can occur in the systole, but more commonly presents in the diastole (22). The majority of IBDMs are asymptomatic despite this dysfunction, and the observed septal wall hypertrophy is typically transient (1-4). In the current study, septal wall thickness was not different between the three groups at 1 month of age, but global and SLSs at the apex and mid-level of the septum were significantly lower in the $\mathrm{OB}+\mathrm{DM}$ group compared with that in the $\mathrm{OB}$ and Lean groups. Diastolic strain rate measures were also decreased in the $\mathrm{OB}+\mathrm{DM}$ group compared with those in the $\mathrm{OB}$ and Lean groups, with preserved systolic strain rate measures. Wang et al. (30) found similar significantly decreased longitudinal strain in the apical segments of the septum in fetuses of women with DM.

Interventricular septal wall thickening and dysfunction in IDBMs may transiently disrupt the performance of both the LV and RV in the early neonatal period (31). In this study, LV morphology was not different among groups; however, at 1 month of age both septal and LV GLSs were lower in OB + DM infants in comparison with those in Lean infants. Biltagi et al. utilized 2DSTE-derived LV deformation imaging to assess LV function in IBDMs in the immediate postnatal period and found reduced LV longitudinal strain and impaired systolic function, but did not distinguish between pre-gestational type 1 and type 2 DM. Previous studies using tissue Doppler and 2DSTE have observed similar decreases in LV GLS in fetuses of obese and DM mothers $(6,30)$. Russel et al. demonstrated that in fetuses of pre-gestational diabetic mothers altered cardiac function (detected by decreased strain imaging) is evident before cardiac structural changes (32). Combined with our results, these studies may suggest that altered cardiac function not only precedes cardiac structural changes but also persists from gestation through the early postnatal period, even after the morphological alterations resolve.

The impact of IBDM on LV diastolic function is unclear from the current literature. $(5,31,33)$ Weber et al. found normal LV early/late-velocity ratio in the fetuses and neonates of well-controlled pre-gestational diabetic mothers (33). Kozak-Barany et al. (31) and Al-Biltagi et al. (5) found that LV diastolic function was significantly impaired in infants in the first week of life, even in the presence of well-controlled glycemia (31). In our study LV diastolic function, as measured by early and late strain rate, was preserved at 1 month of age. Although there may be a component of LV diastolic dysfunction in the early transitional period that reflects the abnormal myocardial relaxation and slower LV filling, the resolution of morphological abnormalities appears to be followed closely by the normalization of LV diastolic function.

Deformation imaging by 2DSTE has also been shown to have a greater sensitivity for measuring global and regional LV function compared with conventional echocardiography in neonates (14). In the first week of life, studies have shown a decrease in shortening fraction in infants born to mothers with pre-gestational and gestational DM (5), whereas other studies have demonstrated normal ejection fraction in gestational diabetes (34). In this study, we found decreased LV GLS and SLS with preserved ejection fraction and shortening fraction in infants born to $\mathrm{OB}+\mathrm{DM}$ mothers at 1 month of age. The clinical significance of these results lie in the notion that advances in neonatal cardiac imaging with 2DSTE now provide the capability of obtaining quantitative information that often supersedes the qualitative information from conventional methods, and permit a more comprehensive (regional and global) assessment of LV function in neonates that could not be previously obtained (14).

Pre-existing maternal diabetes alters both LV and septal performance and potentially complicates $\mathrm{RV}$ function as well (30). There is a paucity of studies $(6,30)$ that have characterized RV function with deformation imaging by 2DSTE in the fetuses of pre-gestational DM mothers, but none in IBDMs. Our study in neonates showed that RV GLS and early diastolic strain rate were significantly decreased in $\mathrm{OB}+\mathrm{DM}$ infants, which is a similar finding to the reported altered RV deformation values in fetuses of mothers with gestational diabetes (30). There are a few mechanisms that could explain RV dysfunction in IBDMs. The pathways that are most responsible for RV remodeling in IBDM likely include insulin resistance and amplified sympathetic nervous and renin-angiotensin-aldosterone systems (26,35-37). The common link between the pathways is the accumulation of collagen in the myocardium and development of myocardial fibrosis, which likely results from prolonged hyperglycemia with advanced glycation end products and excessive production of oxygen free radicals (37). These changes may lead to a loss of elasticity with an increase in ventricular stiffness that first causes diastolic dysfunction, then hypertrophy, and finally systolic dysfunction $(26,35)$. In our study, we observed the following: (i) diastolic dysfunction, as manifested by decreased RV GLS rate at early diastole; (ii) slightly altered RV morphology (increased RV major); and (iii) subsequent systolic dysfunction seen through decreased RV GLS in IBDM.

The strongest potential cause for the deterioration of RV function may be the "interventricular interaction" achieved through the septum (35). We demonstrated that the apexto-base gradient in the RV-free wall longitudinal strain was preserved between the three groups, but that RV GLS was significantly decreased in the OB+DM group. The RV GLS 
measurement includes a part of the septal wall from the apical four-chamber view, further supporting the significance of this interventricular dependence (35).

This study has important limitations. There is evidence that the fetus of a mother with pre-gestational type 2 DM has decreased myocardial deformation in the longitudinal, radial, and circumferential direction, suggesting a diffused pattern of myocardial involvement (6). In this study we evaluated only longitudinal strain imaging, as the feasibility and reproducibility of radial and circumferential strain have not been well described in neonates, and GLS remains the most reliable quantitative tool of the three to assess ventricular function in infants (15). We did not assess torsional mechanics at 1 month of age, but recognize the utility of this emerging speckle tracking modality to assess ventricular mechanics $(5,34)$.

We chose to study the neonates at 1 month of age, rather than at birth, to avoid the early postnatal period of cardiopulmonary instability. Our group has previously developed a standardized cardiac strain imaging protocol to limit the variability in image acquisition (15). Although scanrescan analysis was not performed in this study, one dedicated sonographer, experienced in conventional and quantitative neonatal echocardiography, acquired all of the images, further decreasing the variability in image acquisition. Our study was also limited by the small sample size in each group; however, a figure of 20 neonates was determined to be enough to detect group differences in the outcome variables. Although the current literature does not yet support a link between altered septal function at 1 month of age and adult ventricular dysfunction, future longitudinal studies are needed to follow a larger cohort with serial echocardiograms from early gestation to birth, through the late neonatal period, and into childhood to further investigate these associations and questions.

\section{CONCLUSION}

In conclusion, infants born to obese women with pregestational type $2 \mathrm{DM}$ have reduced interventricular septal wall function at 1 month of age in the absence of septal hypertrophy, which is associated with altered maternal and infant lipid and glucose metabolism. In addition, LV and RV functions are decreased. These findings suggest that maternal obesity and DM may have a prolonged impact on the cardiovascular health of their offspring, despite resolution of cardiac hypertrophy. Aggressive maternal glucose and lipid metabolism management during pregnancy in women with diabetes to modulate infant cardiac dysfunction might be warranted.

\section{STATEMENT OF FINANCIAL SUPPORT}

This research has received funding from the Thrasher Research Fund, the American Diabetes Association, and the National Institutes of Health (NIH) (P30DK056341, P60DK020579, P41GM103422, and UL1RR024992 from the National Center for Research Resources and NIH Roadmap for Medical Research).
Disclosure: The authors declare no conflict of interest.

\section{REFERENCES}

1. Miller HC. Cardiac hypertrophy and extra medullary hematopoesis in newborn infants of prediabetic mothers. Am J Med Sci 1945;209:447.

2. Way GL, Wolfe RR, Eshaghpour E, Bender RL, Jaffe RB, Ruttenberg HD. The natural history of hypertrophic cardiomyopathy in infants of diabetic mothers. J Pediatr 1979;95:1020-5.

3. Halliday HL. Hypertrophic cardiomyopathy in infants of poorlycontrolled diabetic mothers. Arch Dis Child 1981;56:258-63.

4. Trowitzsch E, Bigalke U, Gisbertz R, Kallfelz HC. Echocardiographic profile of infants of diabetic mothers. Eur J Pediatr 1983;140:311-5.

5. Al-Biltagi M, Tolba OA, Rowisha MA, Mahfouz Ael S, Elewa MA. Speckle tracking and myocardial tissue imaging in infant of diabetic mother with gestational and pregestational diabetes. Pediatr Cardiol 2015;36:445-53.

6. Kulkarni A, Li L, Craft M, et al. Fetal myocardial deformation in maternal diabetes mellitus and obesity. Ultrasound Obstet Gynecol 2016.

7. Cooper MJ, Enderlein MA, Tarnoff H, Roge CL. Asymmetric septal hypertrophy in infants of diabetic mothers. Fetal echocardiography and the impact of maternal diabetic control. Am J Dis Child 1992;146: 226-9.

8. Mace S, Hirschfield SS, Riggs T, Fanaroff AA, Merkatz IR. Echocardiographic abnormalities in infants of diabetic mothers. J Pediatr 1979;95: 1013-9.

9. Sheehan PQ, Rowland TW, Shah BL, McGravey VJ, Reiter EO. Maternal diabetic control and hypertrophic cardiomyopathy in infants of diabetic mothers. Clin Pediatr (Phila) 1986;25:266-71.

10. Ece I, Uner A, Balli S, Kibar AE, Oflaz MB, Kurdoglu M. The effects of pre-pregnancy obesity on fetal cardiac functions. Pediatr Cardiol 2014;35: 838-43.

11. Knopp RH, Magee MS, Walden CE, Bonet B, Benedetti TJ. Prediction of infant birth weight by GDM screening tests. Importance of plasma triglyceride. Diabetes Care 1992;15:1605-3.

12. Son GH, Kwon JY, Kim YH, Park YW. Maternal serum triglycerides as predictive factors for large-for-gestational age newborns in women with gestational diabetes mellitus. Acta Obstet Gynecol Scand 2010;89:700-4.

13. Cade WT, Tinius RA, Reeds DN, Patterson BW, Cahill AG. Maternal glucose and fatty acid kinetics and infant birth weight in obese women with type 2 diabetes. Diabetes 2016;65:893-901.

14. Breatnach CR, Levy PT, James AT, Franklin O, El-Khuffash A. Novel echocardiography methods in the functional assessment of the newborn heart. Neonatology 2016;110:248-60.

15. Levy PT, Holland MR, Sekarski T, Hamvas A, Singh GK. Feasibility and reproducibility of right ventricular strain measurements by speckle tracking echocardiography. J Am Soc Echocardiogr 2013;26:1201-3.

16. Lang RM, Badano LP, Mor-Avi V, et al. Recommendations for cardiac chamber quantification by echocardiography in adults: an update from the American Society of Echocardiography and the European Association of Cardiovascular Imaging. J Am Soc Echocardiogr 2015;28:1-39.

17. Singh GK, Cupps B, Pasque M, Woodard PK, Holland MR, Ludomirsky A. Accuracy and reproducibility of strain by speckle tracking in pediatric subjects with normal heart and single ventricular physiology: a twodimensional speckle-tracking echocardiography and magnetic resonance imaging correlative study. J Am Soc Echocardiogr 2010;23:1143-52.

18. Devereux RB, Alonso DR, Lutas EM, et al. Echocardiographic assessment of left ventricular hypertrophy: comparison to necropsy findings. Am J Cardiol 1986;57:450-8.

19. Lopez L, Colan SD, Frommelt PC, et al. Recommendations for quantification methods during the performance of a pediatric echocardiogram: a report from the Pediatric Measurements Writing Group of the American Society of Echocardiography Pediatric and Congenital Heart Disease Council. J Am Soc Echocardiogr 2010;23:465-95.

20. Mertens L, Seri I, Marek J, et al. Targeted Neonatal Echocardiography in the Neonatal Intensive Care Unit: practice guidelines and recommendations for training. Writing Group of the American Society of Echocardiography (ASE) in collaboration with the European Association of 


\section{Cardiac dysfunction in infants of DM $\quad$ Articles}

Echocardiography (EAE) and the Association for European Pediatric Cardiologists (AEPC). J Am Soc Echocardiogr 2011;24:1057-78.

21. Sanchez AA, Levy PT, Sekarski TJ, Hamvas A, Holland MR, Singh GK. Effects of frame rate on two-dimensional speckle tracking-derived measurements of myocardial deformation in premature infants. Echocardiography 2015;32:839-47.

22. Levy PT, Machefsky A, Sanchez AA, et al. Reference ranges of left ventricular strain measures by two-dimensional speckle-tracking echocardiography in children: a systematic review and meta-analysis. J Am Soc Echocardiogr 2016;29:209-5.

23. Voigt JU, Pedrizzetti G, Lysyansky P, et al. Definitions for a common standard for 2D speckle tracking echocardiography: consensus document of the EACVI/ASE/Industry Task Force to Standardize Deformation Imaging. J Am Soc Echocardiogr 2015;28:183-93.

24. Levy PT, El-Khuffash A, Patel MD, et al. Maturational patterns of systolic ventricular deformation mechanics by two-dimensional speckle tracking echocardiography in preterm infants over the first year of age. J Am Soc Echocardiogr 2017 (doi:10.1016/j.echo.2017.03.003; e-pub ahead of print).

25. Cade WT, Reeds DN, Mittendorfer B, et al. Blunted lipolysis and fatty acid oxidation during moderate exercise in HIV-infected subjects taking HAART. Am J Physiol Endocrinol Metab 2007;292:E812-9.

26. Young ME. Adaptation and maladaptation of the heart in diabetes: part ii: potential mechanisms. Circulation 2002;105:1861-70.

27. Schulze PC, Drosatos K, Goldberg IJ. Lipid use and misuse by the heart. Circ Res 2016;118:1736-51.

28. Pauliks LB. The effect of pregestational diabetes on fetal heart function. Expert Rev Cardiovasc Ther 2015;13:67-74.
29. Hornberger LK. Maternal diabetes and the fetal heart. Heart 2006;92: 1019-21.

30. Wang H, Xu Y, Fu J, Huang L. Evaluation of the regional ventricular systolic function by two-dimensional strain echocardiography in gestational diabetes mellitus (GDM) fetuses with good glycemic control. J Matern Fetal Neonatal Med 2015;28:2150-4.

31. Kozák-Bárány A, Jokinen E, Kero P, Tuominen J, Rönnemaa T, Välimäki I. Impaired left ventricular diastolic function in newborn infants of mothers with pregestational or gestational diabetes with good glycemic control. Early Hum Dev 2004;77:13-22.

32. Russell NE, Foley M, Kinsley BT, Firth RG, Coffey M, McAuliffe FM. Effect of pregestational diabetes mellitus on fetal cardiac function and structure. Am J Obstet Gynecol 2008;199:e1-7.

33. Weber HS, Botti JJ, Baylen BG. Sequential longitudinal evaluation of cardiac growth and ventricular diastolic filling in fetuses of well controlled diabetic mothers. Pediatr Cardiol 1994;15:184-9.

34. Liao WQ, Zhou HY, Chen GC, Zou M, Lv X. Left ventricular function in newborn infants of mothers with gestational diabetes mellitus. Zhongguo Dang Dai Er Ke Za Zhi 2012;14:575-7.

35. Tadic M, Cuspidi C, Vukomanovic V, et al. The influence of type 2 diabetes and arterial hypertension on right ventricular layer-specific mechanics. Acta Diabetol 2016;53:791-7.

36. Tadic M, Ivanovic B, Cuspidi C. Metabolic syndrome and right ventricle: an updated review. Eur J Intern Med 2013;24:608-16.

37. Rosen P, Du X, Tschope D. Role of oxygen derived radicals for vascular dysfunction in the diabetic heart: prevention by alpha-tocopherol? Mol Cell Biochem 1998;188:103-1. 